\title{
IMPROVEMENTS IN LIDAR BATHYMETRY DATA ANALYSIS
}

\author{
Hans-Gerd Maas ${ }^{1}$, David Mader ${ }^{1}$, Katja Richter ${ }^{1}$, Patrick Westfeld ${ }^{2}$ \\ ${ }^{1}$ Institute of Photogrammetry and Remote Sensing, Technische Universitat Dresden, Germany \\ (hans-gerd.maas, katja.richter1, david.mader)@tu-dresden.de \\ ${ }^{2}$ Federal Maritime and Hydrographic Agency (BSH), Section Geodetic-hydrographic Techniques and Systems, Germany \\ (patrick.westfeld@bsh.de)
}

Commission II, WGII/9

KEY WORDS: Lidar bathymetry, geometric modelling, waveform analysis

\begin{abstract}
:
Airborne Lidar Bathymetry is a laser scanning technique to measure waterbody bottom topography in shallow waterbodies with limited turbidity. The topic has recently gained relevance due to the advent of new sensor technologies allowing for much higher spatial resolution in bathymetry data capture and due to guidelines demanding regular monitoring of waterbodies. In our contribution, we focus on three important aspects of lidar bathymetry: In the first part, systematic effects of wave patterns will be analysed in order to derive waterbody coordinate correction terms. In the second part, we will apply waveform-stacking techniques to enhance the detectability of water bottom points in lidar bathymetry full waveform signals. In the third part, a dedicated full waveform analysis procedure is shown, which allows for deriving turbidity information from the decay of the signal intensity in the waterbody.
\end{abstract}

\section{INTRODUCTION}

Airborne Lidar Bathymetry (ALB - also called airborne laserscanning bathymetry, airborne lidar hydrography or hydrographic laser in the literature) is an airborne laser scanning technique to determine water bottom topography in shallow clear waters of max. ca. 50 meter depth. It is complementary to established sonar techniques in the sense that sonar reaches much larger depths, but is rather inefficient in shallow water due to the water depth dependency on the water bottom swath width (see e.g. Niemeyer and Sörgel 2013 for an overview). Airborne Lidar Bathymetry has largely replaced attempts of photo-bathymetry, where airborne stereo images of underwater scenes are processed, e.g. Okamoto 1982), due its higher depth range, automation, precision and reliability potential. ALB sensor development over the past years (e.g. Mandlburger, 2018) is largely characterized by a strong increase of the pulse rate and a transition from wide to narrower beam divergence.

Special emphasis has to be put on geometric aspects of refraction, where different degrees of complexity can be imagined for modelling the intersection of a finite diameter laser pulse with a wave-affected non-planar instationary water surface. In addition, full waveform analysis of the returning laser pulse echo is gaining importance in order to reliably and precisely derive water surface and water bottom heights. Figure 1 shows a schematic waveform of a digitized laser pulse echo, composed by the water surface pulse echo, a signal intensity decay caused by turbidity in the water column and the actual water bottom pulse echo.

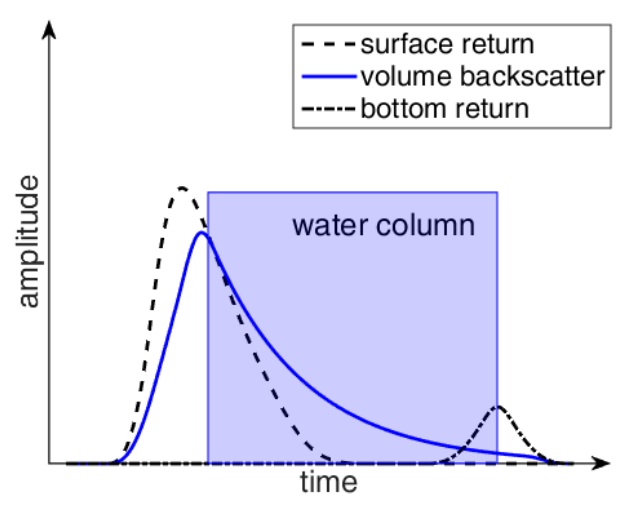

Figure 1: Waveform of a prototype ALB laser pulse echo (Richter et al., 2017)

In reality, ALB laser pulse echoes waveforms often deviate from such a prototype representation. Especially the waterbody bottom echo is often rather weak (and thus hardly detectable) as a consequence of a strong signal intensity loss caused by water turbidity.

In the following chapters, we will address these two aspects of ALB data processing. In chapter 2 we analyze systematic effects of wave patterns on the planimetric and height coordinates determined by ALB. In chapter 3 we apply waveform-stacking techniques to enhance the detectability of water bottom points in lidar bathymetry full waveform signals. In addition, we address a full waveform analysis procedure, which allows for deriving turbidity information from the decay of the signal intensity in the waterbody, in chapter 4 . Methods and results 
will only be addressed briefly here, we refer to the literature for a more detailed description.

\section{CORRECTION OF WAVE PATTERN INDUCED SYSTEMATIC BOTTOM POINT COORDINATE ERRORS}

Geometric modelling in airborne lidar bathymetry is significantly more complex than in conventional airborne laser scanning due to the necessity of modelling refraction effects of the laser pulse passing the air-water interface and due to an increased dispersion of the pulse under water (Figure 2).

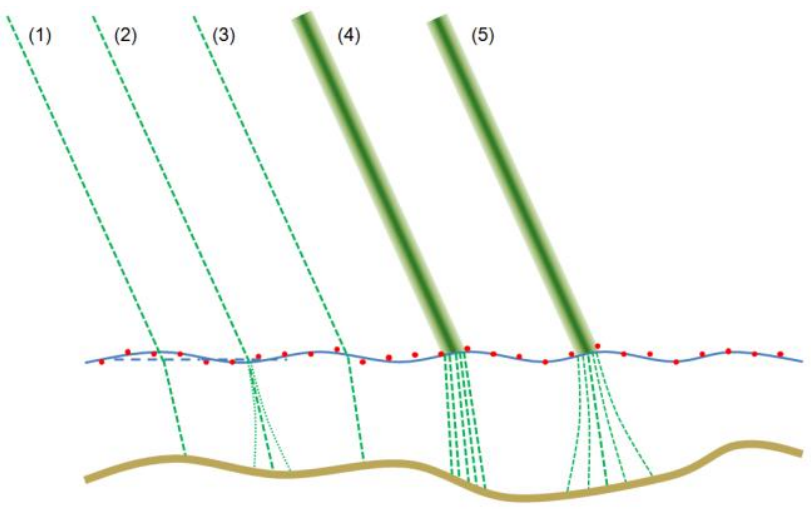

Figure 2: Different representations of the laser beam - water surface interaction (Westfeld et al., 2017)

In the most simple case, the water surface is assumed as a horizontal plane with a height derived from the digitized laser pulse echo waveform. Then Snell's Law can be applied in order to determine the direction of the refracted beam under water, and the reduced speed of light under water (ca. $225.000 \mathrm{~km} / \mathrm{s}$ ) can be applied in depth calculation. The presence of waves has a significant effect on geometric modelling in ALB. Waves change the height of the air-water interface, thus changing the balance between the laser pulse path lengths in air and water. Much stronger is the effect of the tilted surface at wave slopes: These affect the incidence angle and thus also the angle of refraction in Snell's Law, deviating the water bottom point from the correct location linearly with water depth. As a consequence, waves have a very significant effect on both the planimetry and depth coordinates of underwater topography $3 \mathrm{D}$ point cloud coordinates. The effect obviously increases with the height and steepness of waves and with water depth. While the effects of waves were partly averaged out in older wide footprint ALB systems, they lead to a more significant degradation of accuracy for recent small footprint ALB systems.

Therefore, more complex correction methods try to consider the actual water surface geometry. For this purpose, some system providers apply a local water surface tilt based on the intersection of the incident laser ray with a triangular mesh of detected water surface points in their bathymetry data processing methods (Ullrich and Pfennigbauer, 2011). If such a linear local tilt has been considered in data processing, the effects of wave patterns on coordinate accuracy will be reduced. However, besides the simplifications concerning the water surface geometry, both correction approaches are limited by the fact that the laser ray is considered to be an infinitesimal small line only. Effects caused by a finite diameter laser pulse penetrating a curved surface are neglected.

We developed a strict geometric-stochastic model for considering wave effects in lidar bathymetry data processing on the basis of spatio-temporal parameterized water surface models, which allow for a differential modelling of the refracted finite diameter laser pulse path (Westfeld et al., 2017). The model considers the refraction of a finite diameter laser pulse with a wavy water surface. The refraction of a finite diameter laser pulse at a wavy water surface will strongly change the beam profile of the laser pulse. In order to model this effect, a finite diameter laser pulse with a given footprint size is split into a large number of infinitesimally thin sub-pulses, which can then be traced precisely using their local incidence angle onto the water surface and calculation refraction according Snell's Law (Figure 3). Then the center (or centroid) of the locations of the sub-pulses on the bottom can be calculated, using the Gaussian beam profile of the laser pulse in air as subpulse weight information (Figure 4).

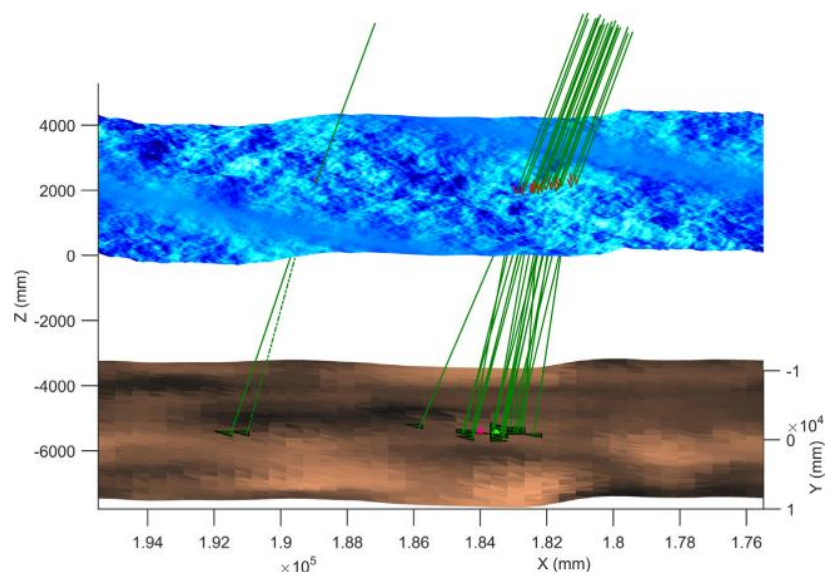

Figure 3: Interaction of an infinitesimally thin (left) and a finite diameter laser pulse (right) with a simulated wave pattern, effects on bottom point determination (Westfeld et al., 2017)

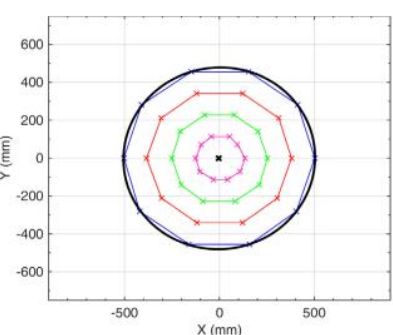

(a)

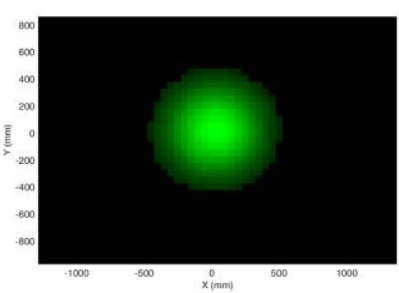

(c)

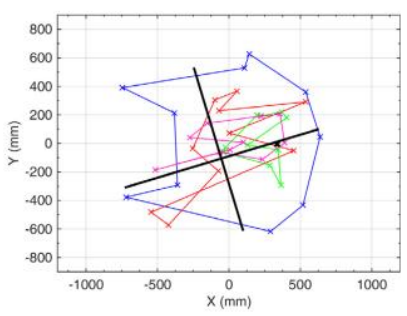

(b)

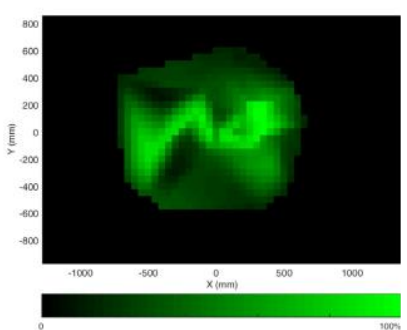

(d)
Figure 4: Wave-induced changes in laser spot size and shape on a simulated wave pattern - left water surface, right water bottom (Westfeld et al., 2017) 
The model can be used to calculate a refraction effect correction for each laser pulse, if the water surface shape can be derived from the data at an appropriate resolution. Strictly modelling a non-planar instationary water surface is, however, a rather complex task, which is not solved here.

However, it can be shown that - in addition to local wavepattern dependent effects on every single laser pulse - wave patterns also have a systematic effect on the waterbody bottom coordinates. In (Westfeld et al., 2017), these effects were quantified on the basis of sequences of water surface simulations using the Tessendorf model (Tessendorf, 2001), allowing for the definition of sea-state dependent correction terms in cases where precise actual water surface modelling is not possible due to the lack of suitable water surface data. The results unveil systematic errors of high resolution lidar bathymetry water bottom coordinates, which may reach several decimetres in the depth coordinate up to meters for the planimetry coordinates. Based on the results of the simulations, correction terms can be derived for typical sea states, thus significantly improving the overall accuracy potential of airborne lidar bathymetry.

\section{IMPROVING LIDAR BATHYMETRY WATER BOTTOM POINT DETECTION BY WAVEFORM STACKING}

Lidar bathymetry systems usually come with full-waveform digitization, allowing to detect water surface and water bottom points by waveform analysis. Depending on the signal quality, which is strongly affected by water turbidity, especially the water bottom point signal in the waveform may be rather weak. As a consequence, the detection of the signal peak representing the water bottom - and thus also depth coordinate determination becomes unreliable.

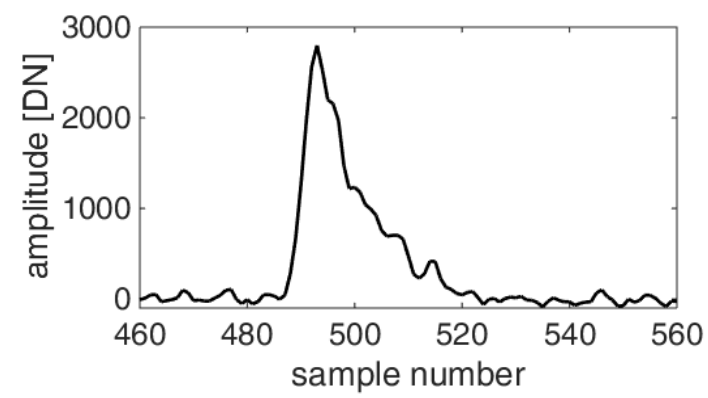

Figure 5: Example of a digitized waveform with poor bottom point echo re cognizability (Richter et al., 2017)

One way to improve the signal-to-noise ratio herein is a neighbourhood dependent consideration. This may for instance be realized by waveform-stacking (e.g. Roncat and Mandlburger, 2016). We developed a waveform-stacking approach analysing the consistency of neighbouring waveforms in order to improve the reliability of water bottom point detection (Mader et al., 2019, see Figure 6). Rather than simply aggregating neighbouring waveforms to a stacked waveform (and thus implicitly lowpass-filtering the water bottom topography model), a voting scheme is realized, wherein small detected peaks in the waveform to be analyzed are only accepted, if the neighbouring waveforms also give evidence for that peak. The vertical alignment of the waveforms is performed via the detected water surface points, and a water bottom tilt can be considered by a vertical tolerance range.

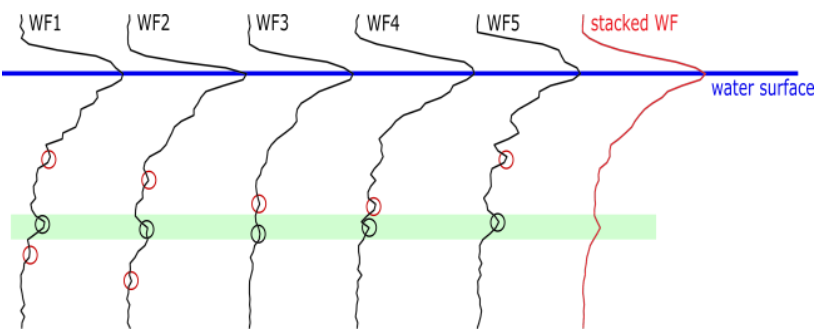

Figure 6: Basic principle of waveform stacking applied to ALB pulse echo waveforms (Mader et al., 2019)

As a result of applying the waveform-stacking technique, a large number of extra points could be extracted, significantly increasing the achievable depth range under given conditions. In a practical test using a dataset of a relatively turbid river, $26 \%$ extra points could be extracted on top of the points delivered by the system manufacturer data processing software (Figure 7).

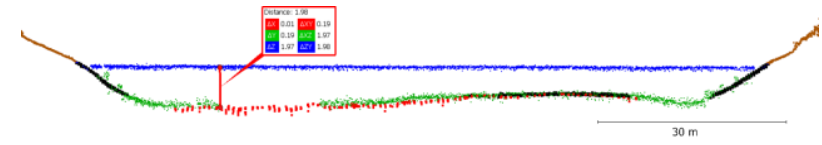

Figure 7: Example of a riverbed profile with water surface points (blue), water bottom points from standard data processing performed by the ALB data provider (black), additional points obtained from waveform stacking (green) and sonar reference measurements (red)

\section{OBTAINING WATER TURBIDITY INFORMATION FROM LIDAR BATHYMETRY FULL WAVEFORM ANALYSIS}

As outlined in chapter 3, underwater laser pulse propagation in lidar bathymetry is characterized by water turbidity dependent attenuation, deteriorating the signal-to-noise-ratio in the digitized laser pulse echo and thus limiting the detection of waterbody bottom points. Inversely, the decay of the laser pulse echo signal in the water column may provide information on water turbidity. Following this idea, the laser pulse echo signal, which is represented in the digitized laser pulse echo waveform, can be analyzed with the goal of deriving parameters describing water turbidity. For that purpose, the decay of each laser pulse echo is modelled by an exponential function:

$$
f(x)=a \cdot e^{(-k \cdot x)}
$$

The decay coefficient $k$ obtained by fitting the exponential function into the part of a digitized waveform data representing the water column depicts an integral measure describing the turbidity in the water column passed by a laser pulse. By fitting an exponential function into each digitized waveform an individual decay coefficient $k$ is determined for each laser pulse echo. This field of decay coefficient depicts a turbidity map. Thus, in contrast to conventional interactive point-wise techniques measuring water turbidity (such as Secchi disk measurements), the approach allows for an automatic full-field characterization. 

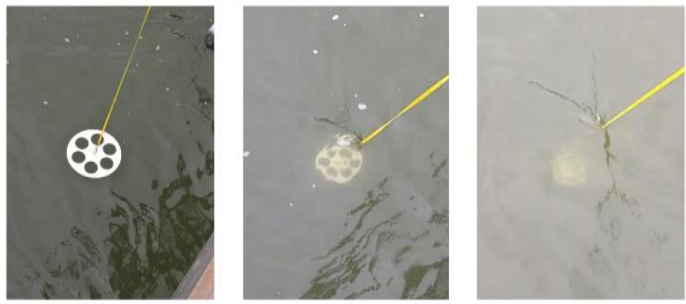

Figure 8: Estimation of visibility depth by lowering a Secchidisk into the water body

The developed technique was applied to a dataset acquired with an AHAB Chiroptera ALB instrument over a shallow inland water, and the results were validated by conventional point-wise turbidity measurement techniques (Richter et al., 2017). The study area (Figure 9) is located at the river Elbe in Germany, close to the city of Klöden. It contains several water bodies, including the rather turbid river Elbe (main channel) and some standing backwater channels with varying water quality and turbidity.

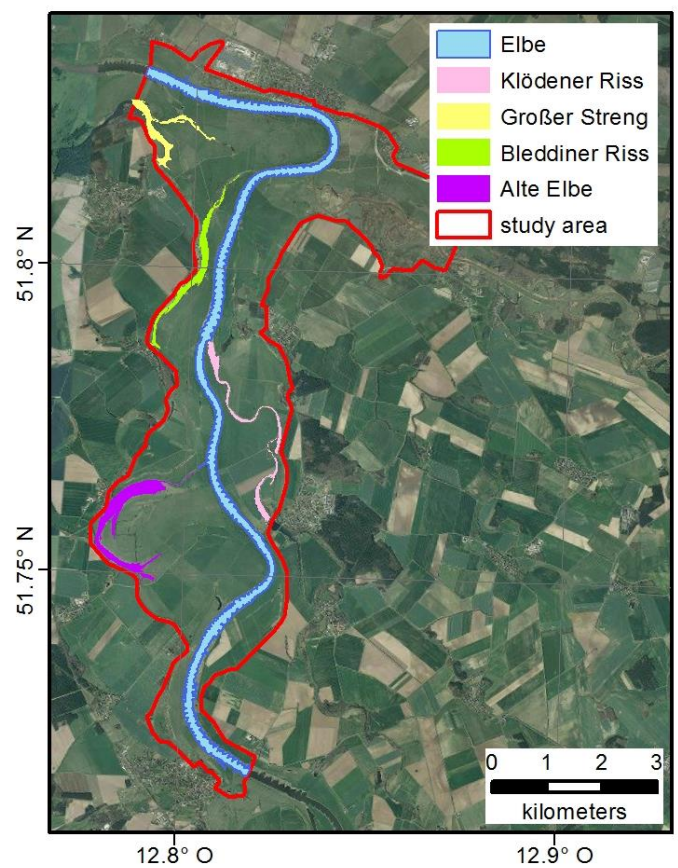

Figure 9: Study area at the river Elbe, Germany (Richter et al., 2017)

Figure 10 shows a turbidity map visualizing the decay coefficients obtained by applying the method as described above for a section of the study area (cmp. Figure 9 ). The colorcoded coefficients are dimension-less and (like Secchi-disk measurements as well) must be calibrated in order to obtain physically meaningful parameters describing turbidity.
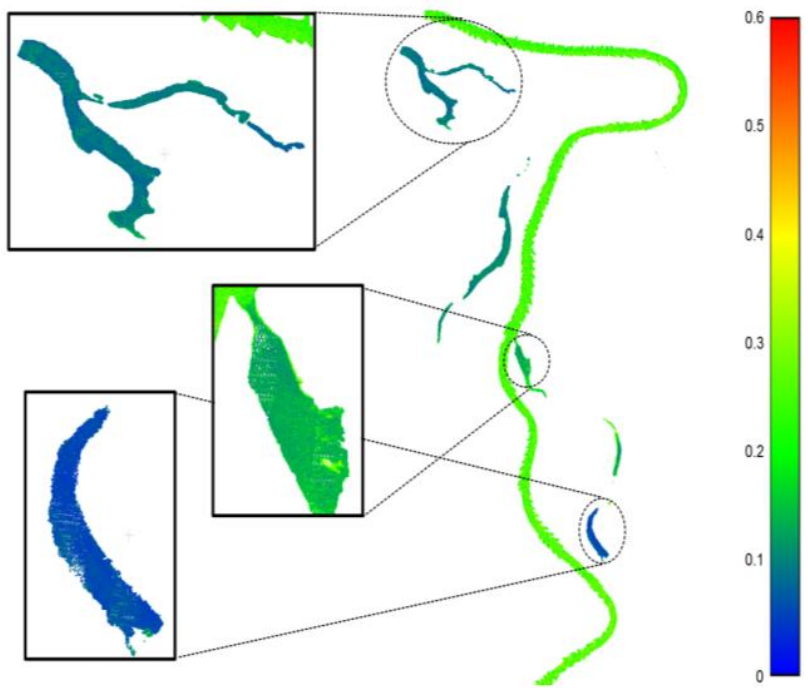

Figure 10: Turbidity map of a section of the river Elbe, Germany (Richter et al., 2017)

Figure 11 shows a comparison of the results for a section of the river Elbe in the same area obtained from processing ALB flight data acquired on two consecutive days. A clear decrease of the decay coefficients (and thus turbidity) between those two days can be seen from the data.
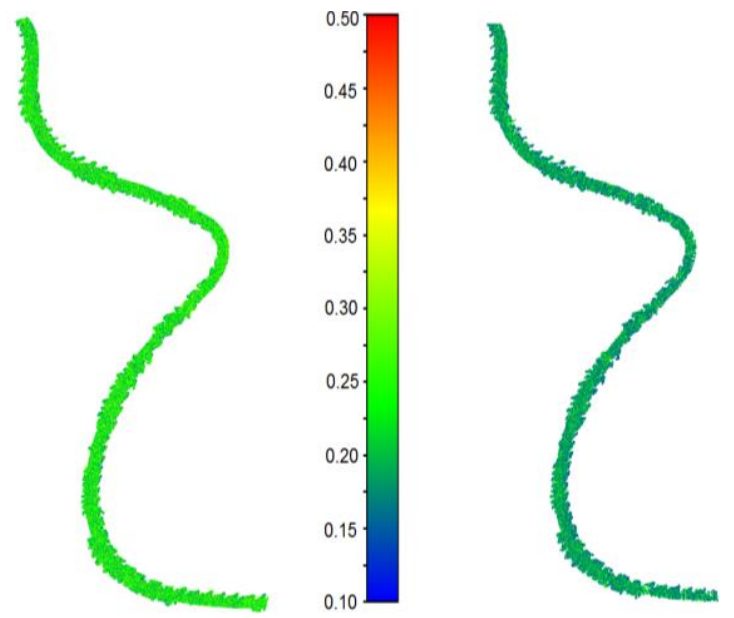

Figure 11: Turbidity map of a section of the river Elbe at two consecutive days (Richter et al., 2017)

\section{ACKNOWLEDGEMENT}

The work presented in this paper was supported by the German Research Foundation (DFG) and by the German Federal Institute of Hydrology (BfG). We would also like to thank the Waterways and Shipping Office Dresden (WSA) for their local support. 


\section{REFERENCES}

Mader, D., Richter, K., Westfeld, P., Weiß, R., Maas, H.-G., 2019: Detektion und Extraktion von Gewässersohlenpunkten aus Laserbathymetriedaten unter Nutzung von Full-WaveformStacking. 39. Jahrestagung der DGPF - Publikationen der DGPF, DGPF Vol. 27

Mandlburger, G., 2018: A review of Airborne Laser Bathymetry sensors. Proc. of the 5th IAHR Europe Congress - New Challenges in Hydraulic Research and Engineering, Eds. Armanini/Nucci, doi:10.3850/978-981-11-2731-1_162-cd

Niemeyer, J., Sörgel, U., 2013: Opportunities of Airborne Laser Bathymetry for the Monitoring of the Sea Bed on the Baltic Sea Coast. International Archives of the Photogrammetry, Remote Sensing and Spatial Information Sciences, Volume XL-7/W2, pp. 179-184

Okamoto, A., 1982: Wave Influences in Two-Media Photogrammetry. Photogrammetric Engineering and Remote Sensing Vol. 48, No. 9, pp. 1487-1499

Richter, K.; Maas, H.-G.; Westfeld, P.; Weiß, R., 2017: An Approach to Determining Turbidity and Correcting for Signal Attenuation in Airborne Lidar Bathymetry. PFG - Journal of Photogrammetry, Remote Sensing and Geoinformation Science, Vol. 85, pp. 1-10

Roncat, A.; Mandlburger, G., 2016: Enhanced detection of water and ground surface in airborne laser bathymetry data using waveform stacking. EGU General Assembly Con-ference Abstracts, Vol. 18, 17016

Secchi, A., 1866: Esperimente per determinare la transparenza del mare. Sul moto ondoso del mare e su le correnti di esso specialmente su quelle littorali, A. Ciadi (ed.), Rome.

Tessendorf, J., 2001. Simulating ocean waters. ACM SIGGraph Course Notes, vol. 47

Ullrich, A., Pfennigbauer, M., 2011. Laserhydrographieverfahren. Patent WO2011137465 A1. Riegl Laser Measurement Systems GmbH

Westfeld, P.; Maas, H.-G.; Richter, K.; Weiß, R., 2017: Analysis and correction of ocean wave pattern induced systematic coordinate errors in airborne LiDAR bathymetry. In: ISPRS Journal of Photogrammetry and Remote Sensing, Vol. 128, pp. 314-325 